\title{
On symplectic uniruling of Hamiltonian fibrations
}

\author{
ClÉMENT HYVRIER
}

\begin{abstract}
Under certain conditions of technical order, we show that closed connected Hamiltonian fibrations over symplectically uniruled manifolds are also symplectically uniruled. As a consequence, we partially extend to nontrivial Hamiltonian fibrations a result of $\mathrm{Lu}$ [14], stating that any trivial symplectic product of two closed symplectic manifolds with one of them being symplectically uniruled verifies the Weinstein Conjecture for closed separating hypersurfaces of contact type. The proof of our result is based on the product formula for Gromov-Witten invariants of Hamiltonian fibrations derived by the author in [5].
\end{abstract}

53D45, 57R17; 55R10

\section{Introduction}

A Hamiltonian fibration is a special case of symplectically fibered space where structure group reduces to the group of Hamiltonian diffeomorphisms of the fiber. Here we will consider closed connected Hamiltonian fibrations over symplectic bases:

$$
\left(F^{2 n_{F}}, \omega\right) \stackrel{\iota}{\hookrightarrow} P^{2 n_{P}} \stackrel{\pi}{\rightarrow}\left(B^{2 n_{B}}, \omega_{B}\right) .
$$

An important feature of such fibrations is that their total spaces admit symplectic structures, denoted by $\omega_{P, \kappa}$, that are compatible with symplectic structures of the fibers (see Section 2.3.1 for more details). In particular, this class of fibrations provides a nice framework to define Gromov-Witten invariants (GW-invariants).

In the present note we are interested in the existence for such fibrations of nonvanishing genus zero Gromov-Witten invariants with a least one point as constraint, namely symplectic uniruledness (see Definition 2.2). In the case the Hamiltonian fibration is rationally cohomologically split ( $c$-split), meaning that the embedding of the fiber induces an injective map in rational cohomology, it is known that symplectic uniruledness of the fiber induces symplectic uniruledness of the total space as shown by Ruan and Li. More precisely:

Theorem 1.1 [12, Proposition 2.10] If $(F, \omega) \stackrel{\iota}{\hookrightarrow}\left(P, \omega_{P, \kappa}\right) \stackrel{\pi}{\rightarrow}\left(B, \omega_{B}\right)$ is a $c$-splitting Hamiltonian fibration, then $\left(P, \omega_{P, \kappa}\right)$ is symplectically uniruled for a class $\sigma \in$ $H_{2}(P ; \mathbb{Z})$ such that $\pi_{*} \sigma=0$ if and only if $(F, \omega)$ is symplectically uniruled. 
In this note we make the following observation, which, in some sense, is complementary to Theorem 1.1:

(O) For c-splitting Hamiltonian fibrations, symplectic uniruledness of the base is sufficient to ensure symplectic uniruledness of the total space.

The $c$-splitting property actually holds in many Hamiltonian fibrations (see Blanchard [1], Hyvrier [5], Kȩdra [6] and Lalonde and McDuff [9]); it is in fact conjectured by Lalonde and McDuff [9] that every Hamiltonian fibration is $c$-split.

Although we think $(\mathbf{O})$ should hold in full generality, we only provide a proof under the following technical assumptions. First, we assume that $(F, \omega)$ is semipositive relative to the total space, ie $(F, \omega)$ verifies condition $(\star)$ described in Section 2.3.2. This condition, verified by Hamiltonian fibrations of real dimension at most six, is a fibered analog of the "standard" semipositivity condition used by McDuff and Salamon [17].

Secondly, we assume that the base is symplectically uniruled for a class $\sigma_{B} \in H_{2}(B ; \mathbb{Z})$ which only admits simple decompositions for some $\omega_{B}$-tame almost complex structure $J_{B}$ on $B$. This condition, made explicit in Definition 2.1, guarantees that, for suitable almost complex structure $J_{P}$ on $P$ lifting $J_{B}$, there are no simple stable $J_{P}$-holomorphic maps in $P$ projecting to a nonsimple stable $J_{B}$-holomorphic maps in $B$ representing $\sigma_{B}$. This is in particular realized by primitive classes admitting pseudo-holomorphic representatives. Here is our main result:

Theorem 1.2 Let $(F, \omega) \stackrel{\iota}{\hookrightarrow}\left(P, \omega_{P, \kappa}\right) \stackrel{\pi}{\rightarrow}\left(B, \omega_{B}\right)$ be a $c$-splitting Hamiltonian fibration. Assume $(F, \omega)$ is semipositive relatively to $P$ and that $\left(B, \omega_{B}\right)$ is symplectically uniruled for some class $\sigma_{B} \in H_{2}(B ; \mathbb{Z})$ admitting only simple decompositions. Then $\left(P, \omega_{P, \kappa}\right)$ is also symplectically uniruled.

This generalizes our result in [5] stating that symplectic uniruling holds for total spaces of Hamiltonian fibrations over symplectically rationally connected base (for a class $\sigma_{B}$ as above), ie when the base has a nonvanishing genus zero Gromov-Witten invariant with a least two points as constraints. However, in that latter case, no $c$-splitting assumption is required.

One of the main ingredients of the proof of Theorem 1.2 is to use fibered almost complex structure on $P$ in order to relate pseudo-holomorphic maps in $P$ with pseudoholomorphic maps in $B$. Roughly speaking, a fibered almost complex structure $J_{P}$ on $P$ is an almost complex structure lifting some $\omega_{B}$-tame almost complex structure $J_{B}$ on $B$, and preserving both horizontal and vertical directions in some splitting of $T P$ induced by a Hamiltonian connection on $P$ (cf Definition 2.3). This geometrical setup 
enables us to relate, generically, some GW-invariant of $P$ with some GW-invariant of $B$ via a product-type formula involving $\mathrm{GW}$-invariants of some Hamiltonian fibration over $S^{2}$. The technical assumptions of the theorem are there to ensure that transversality can be realized within the realm of fibered almost complex structures.

It is believed that the ad hoc hypothesis of Theorem 1.2 can be ruled out using virtual perturbations (see Chen and Li [2], Li and Tian [11] and Ruan [18], amongst others). The problem is to show that one can make perturbations compatibly with the fiber bundle projection $\pi$. Removing these assumptions is part of a joint work in progress with Shengda $\mathrm{Hu}$. In some circumstances however, we can remove the assumption on $\sigma_{B}$. For instance, it can be removed if the base is a smooth projective manifold $\left(B, J_{B}, \omega_{B}\right)$. In such case, Ruan and Kollàr showed that strong symplectic uniruledness is equivalent to projective uniruledness, ie that there is a rational curve passing through every point of $B$. The following proposition then follows from Theorem 1.2.

Corollary 1.3 Let $(F, \omega) \stackrel{\iota}{\hookrightarrow}\left(P, \omega_{P, \kappa}\right) \stackrel{\pi}{\rightarrow}\left(B, \omega_{B}\right)$ be a $c$-splitting Hamiltonian fibration over a smooth projective manifold $\left(B, J_{B}, \omega_{B}\right)$. Assume $(F, \omega)$ is semipositive relatively to $\left(P, \omega_{P, \kappa}\right)$ and that $\left(B, J_{B}, \omega_{B}\right)$ is uniruled. Then $\left(P, \omega_{P, \kappa}\right)$ is strongly symplectically uniruled.

In view of Theorem 1.2 and Theorem 1.1, it is natural to ask the following question: "If neither $F$ or $B$ is symplectically uniruled, can we conclude that $P$ is not symplectically uniruled?" Here is a partial answer:

Theorem 1.4 Assume $\left(B, J_{B}, \omega_{B}\right)$ is a smooth projective manifold and that $P$ is $c$-split. Suppose that neither $\left(B, J_{B}, \omega_{B}\right)$, nor $(F, \omega)$, is symplectically uniruled. Then $\left(P, \omega_{P, \kappa}\right)$ is not symplectically uniruled for any (generic) fibered almost complex structure $J_{P}$ on $P$ lifting $J_{B}$.

Proof Assume by contradiction that $P$ is symplectically uniruled for some class $\sigma \in H_{2}(P ; \mathbb{Z})$. Two possibilities may occur: $\pi_{*} \sigma$ is zero or not. In case $\pi_{*}(\sigma)=0$, we can use the $c$-splitting hypothesis to apply Theorem 1.1 and obtain a contradiction. Now, let's consider the case where $\pi_{*} \sigma \neq 0$. Since $P$ is symplectically uniruled, through every point $p \in P$ there exists a $\sigma$-rational $J_{P}$ pseudo-holomorphic map $u$ passing through $p$. Since $J_{P}$ is fibered, the map $u_{B}:=\pi \circ u$ is $J_{B}$ pseudo-holomorphic. Moreover, $u_{B}$ passes through $\pi(p)$. Consequently, $B$ is covered by rational curves. Since $B$ is projective this implies that $B$ is symplectically uniruled; thus we have a contradiction. 
Before proceeding to the applications, we make a remark concerning possible generalizations of Theorem 1.2, when we replace symplectic uniruledness by $k$-symplectic rational connectedness with $k>1$ (cf Definition 2.2). Roughly speaking, a symplectic manifold is said to be $k$-symplectically rationally connected if there exists a nonvanishing Gromov-Witten invariant with $k$ points as constraints.

It is easy to find examples where Theorem 1.1 and observation $(\mathbf{O})$ do not generalize to $k>1$, for instance when $P$ is a trivial symplectic fibration. Nevertheless, it is possible to combine symplectic rational connectedness of the fiber and of the base in order to obtain symplectic rational connectedness of the total space. Before making a precise statement, recall that the relative semipositivity condition on $(F, \omega)$ implies that the fiber is actually semipositive. It is well-known that under such hypothesis, the quantum homology of $(F, \omega)$ is generically well-defined. This quantum homology is endowed with a ring structure called quantum product, which can be seen as a deformation of the intersection product in homology. We introduce the following notation: let [pt] be the homology class of a point in $F$, we write $[\mathrm{pt}]_{Q}$ to denote the class of the point seen as an element of the Quantum homology of $F$. Also, let $[\mathrm{pt}]_{Q}^{k}$ denote the $k$-th power of $[\mathrm{pt}]_{Q}$ with respect to the quantum product.

Theorem 1.5 Suppose $(F, \omega)$ is semipositive relatively to $\left(P, \omega_{P, \kappa}\right)$, and assume that $[\mathrm{pt}]_{Q}^{l} \neq 0, l>1$. Furthermore, suppose that $\left(B, \omega_{B}\right)$ is $(l+1)$-symplectically rationally connected for some class $\sigma_{B} \in H_{2}(B, \mathbb{Z})$ admitting only simple decompositions. Then $P$ is $l$-symplectically rationally connected.

Theorem 1.2 can be applied to the Weinstein Conjecture for separating hypersurfaces, or in shorter terms the shW-Conjecture. In 1979, A Weinstein [22] conjectured that: "Every closed hypersurface $S$ of contact type in a given symplectic manifold $(X, \omega)$ carries a closed characteristic." Since Viterbo's proof of the conjecture for $\left(\mathbb{R}^{2 n}, \omega_{0}\right)$ in 1986 [21], many results followed. In particular, Hofer and Viterbo highlighted in [3] the strong interplay between genus zero Gromov-Witten invariants and the conjecture, in cases where the hypersurface $S$ separates $X$, ie when there exist submanifolds $X^{+}$ and $X^{-}$of $X$ having common boundary and such that $X=X^{+} \cup X^{-}$and $S=$ $X^{+} \cap X^{-}$. Note that this latter condition is realized whenever $H^{1}\left(X, \mathbb{Z}_{2}\right)=0$. Shortly after, these results were extended by Liu and Tian in [13], to any symplectic manifold and any genus. In 2000, using the results of Liu and Tian, Guangcun Lu proved:

Theorem 1.6 (Lu [14, Corollary 3]) Any separating hypersurface of contact type in a symplectically uniruled closed symplectic manifold $\left(B, \omega_{B}\right)$ admits a closed characteristic. In particular, the shW-Conjecture holds in products $\left(B \times F, \omega_{B} \oplus \omega\right)$ of a symplectically uniruled closed symplectic manifold $\left(B, \omega_{B}\right)$ with any symplectic manifold $(F, \omega)$. 
As a direct consequence of the first assertion of Theorem 1.6 and of Theorem 1.2 we obtain the following generalization to nontrivial Hamiltonian fibrations of the second assertion of Theorem 1.6:

Corollary 1.7 The shW-Conjecture holds in $c$-splitting Hamiltonian fibrations $\left(P, \omega_{P, \kappa}\right)$ with relatively semipositive fiber $(F, \omega)$ and with symplectically uniruled base $\left(B, \omega_{B}\right)$ for some class $\sigma_{B}$ admitting only simple decompositions.

In fact, as it was shown in [5, Corollary], $c$-splitting holds for Hamiltonian fibrations over 2-symplectically rationally connected base for some class $\sigma_{B}$ as in the corollary above. It then follows from Corollary 1.7 that the shW-Conjecture holds in Hamiltonian fibrations with relatively semipositive fiber and with 2 -symplectically rationally connected base for some class admitting only simple decompositions. In particular, the shW-Conjecture holds in Hamiltonian fibrations (with relatively semipositive fiber) over $\left(\mathbb{C} P^{n}, \omega_{F S}\right)$ where $\omega_{F S}$ denotes the Fubini-Study Kähler form, and over $\left(S^{2} \times S^{2}, \omega \oplus \omega\right)$ where $\omega$ is an area form on $S^{2}$. It also holds for $c$-splitting Hamiltonian fibrations over ruled surfaces. Finally it should also hold for $c$-splitting Hamiltonian fibrations over blow-ups of such bases, since symplectic uniruledness is a symplectic cobordism invariant by $\mathrm{Hu}, \mathrm{Li}$ and Ruan [4].

This note is organized as follows: in Section 2 we introduce the notions and notation needed for the proofs of the results, and in Section 3 we proceed with the proofs.

Acknowledgements I would like to thank François Lalonde and Octav Cornea for their useful comments and their encouragements. I would also like to thank François for his constant support and generosity. Finally, I thank Rémi Leclercq for his suggestions and comments on an earlier version of this note, that helped improve the presentation of the paper.

\section{Framework and tools}

\subsection{Gromov-Witten invariants}

Let $J$ be an $\omega$-tame almost complex structure on $\left(X^{2 n_{X}}, \omega\right)$, ie $J$ is a smooth endomorphism of $T X$ such that

$$
J^{2}=-\operatorname{Id}_{T X} \quad \text { and } \quad \omega(v, J v)>0 \text { for all } v \in T X .
$$

The set of all such almost complex structures is nonempty and contractible. We denote by $c_{1}^{T X}$ the associated first Chern class of $T X$, and say that $(X, \omega)$ is semipositive if 
and only if there are no spherical class $A \in H_{2}(X ; \mathbb{Z})$ with positive area $\omega(A)$ such that

$$
3-n_{X} \leq c_{1}^{T X}(A)<0 .
$$

We begin by recalling the definition of genus zero Gromov-Witten invariant for $(X, \omega)$ assuming it is semipositive. We follow the expositions of McDuff and Salamon [17, Chapter 7] or Ruan and Tian [19]. At the end we introduce the definition of simple decomposability.

2.1.1 Definition of Gromov-Witten invariants Recall a genus zero $J$-holomorphic map of $X$ is a smooth map $u: S^{2} \rightarrow X$ solution to the Cauchy-Riemann equation

$$
\bar{\partial}_{J} u:=1 / 2\left(d u+J \circ d u \circ j_{0}\right)=0,
$$

where $j_{0}$ stands for the standard complex structure on $S^{2} \cong \mathbb{C} \cup\{\infty\}$. Roughly speaking, a genus zero Gromov-Witten invariant of $X$ is a count, up to reparametrizations of the domain, of $J$-holomorphic rational maps with marked points with values in $X$, satisfying some prescribed constraints at the marked points. More precisely, consider the moduli space of unparametrized genus zero $J$-holomorphic maps in $X$ with $l$ marked points and representing the class $A$

$$
\begin{aligned}
\mathcal{M}_{0, l}(X, A, J):=\{(u, \mathbf{x}):= & \left(u, x_{1}, \ldots, x_{l}\right) \in C^{\infty}\left(S^{2}, X\right) \times\left(S^{2}\right)^{l} \\
& \left.\mid x_{i} \neq x_{j} \text { if } i \neq j, \bar{\partial}_{J} u=0,\left[u\left(S^{2}\right)\right]=A\right\} / \mathrm{PSL}_{2}(\mathbb{C}) .
\end{aligned}
$$

Let $\overline{\mathcal{M}}_{0, l}(X, A, J)$ denote its compactification, in the sense of Gromov, which coincides with the moduli space of isomorphism classes $[\Sigma, u, \mathbf{x}]$ of stable $J$-holomorphic maps with $l$ marked points that represent $A$. This is a stratified space with finitely many strata. Its top stratum is the subset $\mathcal{M}_{0, l}^{*}(X, A, J) \subset \mathcal{M}_{0, l}(X, A, J)$ consisting of simple $J$-holomorphic maps, ie maps that are somewhere injective. This stratum is, for generic $J$, a naturally oriented manifold with dimension $2 n_{X}+2 c_{1}^{T X}(A)-6+2 l$. There are two natural maps defined on $\overline{\mathcal{M}}_{0, l}(X, A, J)$, namely the evaluation at the marked points map

$$
\mathrm{ev}_{l, J}^{X}: \overline{\mathcal{M}}_{0, l}(X, A, J) \rightarrow X^{l}, \quad\left[\Sigma, u, x_{1}, \ldots, x_{l}\right] \mapsto\left(u\left(x_{1}\right), \ldots, u\left(x_{l}\right)\right),
$$

and, for $l \geq 3$, the forgetful map

$$
\mathfrak{f}: \overline{\mathcal{M}}_{0, l}(X, A, J) \rightarrow \overline{\mathcal{M}}_{0, l}
$$

assigning to every stable map the underlying reduced stable curve. Formally, when $l \geq 3$, Gromov-Witten invariants are the values of a multilinear homomorphism

$$
\langle\cdot\rangle_{A, l}^{X}: H_{*}\left(\overline{\mathcal{M}}_{0, l}, \mathbb{Q}\right) \otimes\left(H_{*}(X, \mathbb{Q})\right)^{\otimes^{l}} \rightarrow \mathbb{Q},
$$


where

$$
\left\langle D ; \beta_{1}, \ldots, \beta_{l}\right\rangle_{A, l}^{X}:=\int_{\overline{\mathcal{M}}_{0, l}(X, A, J)}\left(\operatorname{ev}_{l, J}^{X}\right)^{*}\left(\mathrm{PD}_{X^{l}}\left(\beta_{1} \otimes \cdots \otimes \beta_{l}\right)\right) \cup \mathfrak{f}^{*} \operatorname{PD}_{\overline{\mathcal{M}}_{0, l}}(D),
$$

which is set to be zero unless

$$
2 n_{X}+2 c_{1}^{T X}(A)=\sum_{i=1}^{l}\left(2 n_{X}-\operatorname{deg}\left(\beta_{i}\right)\right)-\operatorname{deg}(D)
$$

When $l<3$, there is no forgetful map and we simply "integrate" the pullback under $\mathrm{ev}_{l, J}^{X}$ of the product of the $\operatorname{PD}\left(\beta_{i}\right)$; in that case we use the notation $\left\langle\beta_{1}, \ldots, \beta_{l}\right\rangle_{A, l}^{X}$, which could be viewed as $\left\langle\left[\overline{\mathcal{M}}_{0, l}\right] ; \beta_{1}, \ldots, \beta_{l}\right\rangle_{A, l}^{X}$ if we consider $\overline{\mathcal{M}}_{0, l}$ as a manifold of negative dimension $2 l-6$.

The integration above has to be understood as evaluation of the cohomology class with the fundamental cycle of $\overline{\mathcal{M}}_{0, l}(X, A, J)$. However, this space may not carry a fundamental class. In fact, lower strata in $\overline{\mathcal{M}}_{0, l}(X, A, J)$ may have dimensions greater than $\mathcal{M}_{0, l}^{*}(X, A, J)$, due to the possible presence of stable maps with multiply covered components. This problem does not show up when the manifold is semipositive. Concretely, this condition imposes that the "boundary component" $\overline{\mathcal{M}}_{0, l}(X, A, J) \backslash \mathcal{M}_{0, l}^{*}(X, A, J)$ is generically of codimension at least two with respect to the top stratum. Then, consider cycles $V_{1}, \ldots, V_{l}$ in $X$ representing the $\beta_{i}$ 's, and a cycle $D$ in $\overline{\mathcal{M}}_{0, l}$ representing $D$. Assume that these cycles are in general position, and such that $\mathfrak{f} \times \mathrm{ev}_{l, J}^{X}$ is strongly transverse to the product $D \times V_{1} \times \cdots \times V_{l}$. The corresponding Gromov-Witten invariant is realized as the intersection number $\left(\mathfrak{f} \times \operatorname{ev}_{l, J}^{X}\right) \cdot\left(D \times V_{1} \times \cdots \times V_{l}\right)$ (which can be seen to be $\mathbb{Z}$-valued in that case). In particular, GW-invariants generically count simple maps.

2.1.2 On the semipositivity assumption We should mention that the semipositivity assumption can be removed using virtual perturbations of the Cauchy-Riemann equation as in Li and Tian [11], Ruan [18] and Ruan and Tian [19], amongst others. However, we will not work in such generality. According to McDuff and Salamon [17] we say that a stable $J$-holomorphic map is simple if and only if it has no nonconstant multiply covered component and no two nonconstant components having the same image in $X$. This leads to the following definition:

Definition 2.1 Let $A \in H_{2}(X ; \mathbb{Z})$ be a spherical class representable by $J$-holomorphic maps. We say that $A$ only admits simple decompositions if every stable $J$-holomorphic map in $\overline{\mathcal{M}}_{0, l}(X, A, J)$ has no nonconstant multiply covered component and no two nonconstant components having the same image in $X$. 
This is what we request from $\sigma_{B}$ in Theorem 1.2. Note that the set of $\omega$-tame almost complex structures $J$ with respect to which a given class $A$ only admits simple decompositions is open in the set of $\omega$-tame almost complex structures. However, we cannot make sure that this set is connected or nonempty.

\subsection{Symplectic rational connectedness}

Using the notation introduced in the preceding paragraph, we define the notion of $k$-symplectic rational connectedness, following $\mathrm{Hu}, \mathrm{Li}$ and $\mathrm{Ruan}$ [4] and $\mathrm{Lu}$ [14].

Definition 2.2 Let $k>0$ be an integer, and let $A \in H_{2}(X ; \mathbb{Z})$ be a spherical homology class. A symplectic manifold $(X, \omega)$ is $k$-symplectically rationally connected for $\sigma$, or simply $k-S R C$ for $\sigma$, if and only if there exist classes $\beta_{k+1}, \ldots, \beta_{l} \in H_{*}(X, \mathbb{Q})$, and $D \in H_{*}\left(\overline{\mathcal{M}}_{0, l}, \mathbb{Z}\right)$ such that

$$
\left\langle D ;[\mathrm{pt}], \ldots,[\mathrm{pt}], \beta_{k+1}, \ldots, \beta_{l}\right\rangle_{A, l}^{X} \neq 0 .
$$

We will say that $(X, \omega)$ is $k$-symplectically rationally connected if there exists $\sigma$ such that it is $k-S R C$ for $\sigma$. If $k=1$, we will say that $(X, \omega)$ is symplectically uniruled or $S U$. Furthermore, if $(X, \omega)$ is symplectically uniruled and $l=3$, we say that $(X, \omega)$ is strongly symplectically uniruled or SSU.

In particular, $(X, \omega)$ is $k$-SRC for $A$ only if, through every $k$ generic points of $X$, there is a genus zero pseudo-holomorphic map representing $A$. The converse may not be true in general. Nevertheless, the equivalence holds in smooth projective varieties.

Theorem 2.1 (Kollàr [8]; Ruan [18]) A smooth projective variety is symplectically uniruled if and only if it is projectively uniruled, ie through every point of the manifold there is a holomorphic map.

Actually, it follows from the splitting axiom for Gromov-Witten invariants that a projective manifold is (projectively) uniruled if and only if it is strongly symplectically uniruled, as pointed out by Ruan [18]. The main ingredient of the proof is the following property of rational curves in projectively uniruled manifolds $(X, J, \omega)$ due to Kollàr, Miyaoka and Mori (see Kollàr [7, Theorem 3.11]): for a very general point $b \in X$, if $u: \mathbb{C} P^{1} \rightarrow X$ is a morphism such that $\left[u\left(\mathbb{C} P^{1}\right)\right] \neq 0$ and $u(0)=b$, then $H^{1}\left(\mathbb{C} P^{1}, u^{*} T X\right)=0$. Such morphism is said to be free (over 0 ). Now, for $b \in X$ and a spherical class $\sigma$, let $\mathcal{M}(X, \sigma, J ; b)$ denote the moduli space of $\sigma$ rational $J$-holomorphic maps $u: \mathbb{C} P^{1} \rightarrow X$ such that $b \in \operatorname{Im}(u)$. We will say that $\sigma$ is free if, for every general point $b \in X$, the moduli space $\mathcal{M}(X, \sigma, J ; b)$ only consists of free morphisms. For our purpose, we mildly refine the statement of Theorem 2.1 by the following straightforward observation. 
Lemma 2.2 If a smooth projective variety $(X, J, \omega)$ is uniruled, it is strongly symplectically uniruled for a free class $\sigma$ admitting only simple decompositions.

Proof Since $X$ is uniruled, for every $b \in X$ there exists a morphism $u$ : $\mathbb{C} P^{1} \rightarrow X$ such that $b \in \operatorname{Im}(u)$. Now, fix a sufficiently general point $b$. In the proof of Theorem 2.1 given in [18], Ruan shows the equality between

$$
\begin{aligned}
& N_{1}:=\min \{\omega(\sigma)>0 \mid \mathcal{M}(X, J, \sigma ; b) \neq \varnothing\}, \\
& N_{2}:=\min \left\{\omega(\sigma)>0 \mid \exists \alpha_{1}, \ldots, \alpha_{l} \in H_{*}(X),\left\langle[\mathrm{pt}] ;[\mathrm{pt}], \alpha_{1}, \ldots, \alpha_{l}\right\rangle_{\sigma, l+1}^{X} \neq 0\right\} .
\end{aligned}
$$

He also points out that $N_{2}$ is realized by classes with nontrivial three point GWinvariant. Suppose $\sigma \in H_{2}(X ; \mathbb{Z})$ realizes this minimum. Then every rational curve passing through $b$ and representing $\sigma$ is irreducible, ie any stable holomorphic map through $b$ is simple. It follows that $\mathcal{M}(X, J, \sigma ; b)$ is compact. Furthermore, by choosing $b$ general enough, for any $u \in \mathcal{M}(X, J, \sigma ; b)$ the obstruction $H^{1}\left(\mathbb{C} P^{1}, u^{*} T X\right)$ vanishes; thus, the moduli space $\mathcal{M}(X, J, \sigma ; b)$ is a smooth oriented manifold, as desired.

\subsection{Hamiltonian fibrations and the product formula}

In this section we introduce the notion of Hamiltonian fibrations which provides natural framework to study Gromov-Witten invariants.

2.3.1 Hamiltonian fibrations By definition, a symplectic fibration is a locally trivial smooth fibration with symplectic reference fiber $(F, \omega)$,

$$
(F, \omega) \stackrel{\iota}{\hookrightarrow} P \stackrel{\pi}{\rightarrow} B,
$$

and which structure group lies in the group of symplectic diffeomorphisms of the fiber, denoted $\operatorname{Symp}(F, \omega)$. It follows that each fiber $F_{b}:=\pi^{-1}(b)$ is naturally equipped with a symplectic form $\omega_{b}$. A symplectic fibration is Hamiltonian if the structure group can be reduced to the group $\operatorname{Ham}(F, \omega)$ of Hamiltonian diffeomorphisms. Hamiltonian fibrations are characterized as follows:

Theorem 2.3 [16, Theorem 6.36] A symplectic fibration $P$ as above is Hamiltonian if and only if the following two conditions are verified:

$\left(H_{1}\right) \quad P$ is symplectically trivial over the 1 -skeleton of $B$.

$\left(H_{2}\right)$ There exists a unique closed connection 2-form $\tau \in \Omega^{2}(P)$ extending the family $\left\{\omega_{b}\right\} b \in B$ such that the integration of $\tau^{n_{F}+1}$ over the fibers of $P$ vanishes. 
The closed 2-form in the theorem above is usually referred to as the coupling form. The coupling form defines a connection on $P$, ie a splitting at each $p \in P$,

$$
T_{p} P=\operatorname{Hor}_{\tau, p} \oplus \operatorname{ker} d \pi(p) .
$$

The theorem states that the corresponding holonomy around any loop in $B$ is in $\operatorname{Ham}(F, \omega)$. Any other closed extension $\tau^{\prime}$ of $\omega$ generating the same horizontal distribution Hor $_{\tau}$ is actually uniquely obtained from $\tau$ via the equation

$$
\tau^{\prime}=\tau+\pi^{*} \varrho, \quad \varrho \in \Omega^{2}(B) .
$$

One of the main features of Hamiltonian fibrations over closed symplectic bases is that they can be given symplectic structures compatibly with the family $\left\{\omega_{b}\right\}_{b \in B}$. Such symplectic structures are obtained as follows:

$$
\omega_{P, \kappa}:=\tau+\kappa \pi^{*} \omega_{B},
$$

where $\kappa>0$ is a large enough real number such that $\omega_{P, \kappa}$ is nondegenerate. We will also use $\tau$ to denote the deRham cohomology class corresponding to $\tau$. There is another canonical cohomology class of $P$ that will play an important part in the next paragraphs, namely the vertical Chern class $c_{v} \in H^{2}(P ; \mathbb{Z})$. This class is defined as the first Chern class of the vertical subbundle $\operatorname{ker} d \pi$.

As a specific example, let us mention the case of Hamiltonian fibrations over $S^{2}$ with fiber $(F, \omega)$, which will play an important part in this note. This class of examples is particularly important in symplectic topology due to the correspondence between these fibrations and the fundamental group of $\operatorname{Ham}(F, \omega)$. More precisely, for $\gamma \in$ $\pi_{1}(\operatorname{Ham}(F, \omega))$ one defines a Hamiltonian bundle $\pi: P_{\gamma} \rightarrow S^{2}$ with fiber $F$ via the clutching construction: choose any representative $\tilde{\gamma}:[0,1] \rightarrow \operatorname{Ham}(F, \omega)$ for $\gamma$, then

$$
P_{\gamma}:=\frac{\left(D^{+} \times F\right) \sqcup\left(D^{-} \times F\right)}{\left(e^{2 \pi i \theta}, x\right) \sim\left(e^{-2 \pi i \theta}, \tilde{\gamma}(\theta) \cdot x\right) \text { on } S^{1} \times F},
$$

where $D^{ \pm} \subset \mathbb{C}$ denotes the closed unit disc. This construction is independent of the representative $\tilde{\gamma}$; moreover, any Hamiltonian fibration over $S^{2}$ with $(F, \omega)$ as fiber can be constructed in this way (see Lalonde, McDuff and Polterovich [10] or Seidel [20]). In this context, the coupling class will be denoted by $\tau_{\gamma}$ and $c_{\gamma}$ will denote the vertical Chern class associated to $P_{\gamma}$.

2.3.2 The product formula Following [5] we give a product formula for GWinvariants of a Hamiltonian fibration, assuming the fiber $(F, \omega)$ is semipositive relative to the total space, ie

$(\star) \quad \forall A \in H_{2}^{S}(F), \quad \omega(A)>0, \quad c^{v}(\iota(A)) \geq 3-n_{P} \Longrightarrow c^{v}(\iota(A)) \geq 0$, 
where $H_{2}^{S}(F)$ is the spherical homology subgroup of $H_{2}(F, \mathbb{Z})$ (ie the image of $\pi_{2}(F)$ under the Hurewicz map) and $\iota$ denotes the map in homology induced from the natural embedding of the fiber. For instance, this implies that the fiber is semipositive. For this purpose we equip $P$ with an (almost) complex structure $J_{P}$ which is compatible with the fibration structure and a Hamiltonian connection in the sense given below:

Definition 2.3 An almost complex structure $J_{P}$ on $P$ is said to be compatible with $\pi$ and $\tau$, or just fibered, if and only if there exists an $\omega_{B}$-tame complex structure $J_{B}$ on $B$ and a family of $\omega_{b}$-tame almost complex structures $J_{b}$ in $F_{b}$ such that

- $d \pi \circ J_{P}=J_{B} \circ d \pi$,

- $J_{b}:=\left.J_{P}\right|_{F_{b}}$ for all $b \in B$,

- $J_{P}$ preserves the horizontal distribution induced by $\tau$.

For fibered $J_{P}$, the projection $\pi$ induces a map between moduli spaces:

$$
\bar{\pi}: \mathcal{M}_{0, l}\left(P, \sigma, J_{P}\right) \rightarrow \mathcal{M}_{0, l}\left(B, \sigma_{B}, J_{B}\right), \quad[\Sigma, u, \mathbf{x}] \mapsto[\Sigma, \pi(u), \mathbf{x}],
$$

where $\sigma_{B}:=\pi_{*} \sigma$. In what follows we assume that $\sigma_{B}$ is nonzero. The fiber of $\bar{\pi}$ over $\left[\Sigma, u_{B}, \mathbf{x}\right]$ can be described as follows. Let $C$ denote the image of $u_{B}$ in $B$, and let $P_{C}$ denote the restriction of $P$ along $C ; P_{C}$ defines a Hamiltonian fibration over $S^{2}$ with coupling form given by the pullback of $\tau$ under the natural inclusion $\iota_{P_{C}}: P_{C} \hookrightarrow P$. If $J_{C}$ denotes the fibered almost complex structure on $P_{C}$ given by the restriction of $J_{P}$ to $P_{C}$ we have the following identification:

$$
\bar{\pi}^{-1}\left(\left[\Sigma, u_{B}, \mathbf{x}\right]\right) \equiv{ }_{B_{\sigma}:=\left\{\sigma^{\prime} \in H_{2}\left(P_{C} ; \mathbb{Z}\right) \mid \iota_{P_{C}} \sigma^{\prime}=\sigma\right\}}\left(\mathcal{M}_{0, l}\left(P_{C}, J_{C}, \sigma^{\prime}\right) \cap \mathfrak{f}^{-1}([\Sigma, \mathbf{x}])\right) .
$$

Regarding evaluation at the marked points maps we have the commutative diagram:

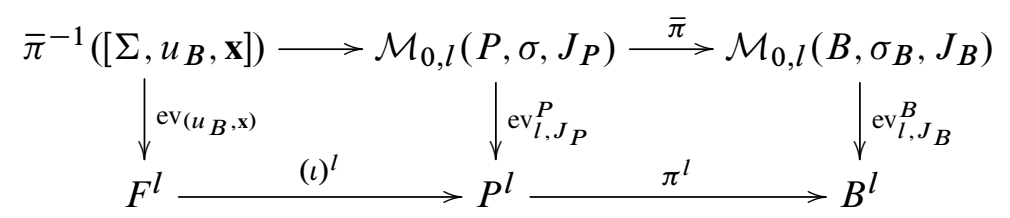

where

$$
\operatorname{ev}_{\left(u_{B}, \mathbf{x}\right)}: \bar{\pi}^{-1}\left(u_{B}, \mathbf{x}\right) \rightarrow F^{l}, \quad u \mapsto\left(u\left(x_{1}\right), \ldots, u\left(x_{l}\right)\right) \in \prod_{i=1}^{l} F_{u_{B}\left(x_{i}\right)} .
$$


The product formula is obtained by considering the (respective) intersections of $\mathrm{ev}_{\left(u_{B}, \mathrm{x}\right)}$, $\mathrm{ev}_{l, J_{B}}^{B}$, and $\mathrm{ev}_{l, J_{P}}^{P}$, with the product cycles:

$$
\mathcal{C}^{F}:=\prod_{i=1}^{l} V_{i}^{F}, \quad \mathcal{C}^{B}:=\prod_{i=1}^{l} V_{i}^{B}, \quad \mathcal{C}^{P}:=\prod_{i=1}^{l} V_{i}^{P},
$$

where $V_{i}^{F}, V_{i}^{B}$ and $V_{i}^{P}$, respectively represent homology classes $c_{i}^{F}, c_{i}^{B}$ and $c_{i}^{P}$, verifying that for some integer $0 \leq m \leq l$ :

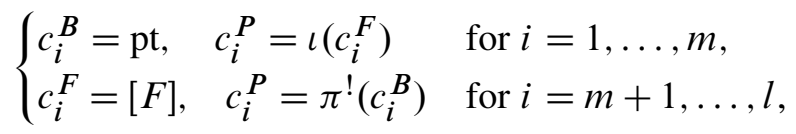

where $\pi^{!}$stands for the shriek map

$$
\pi^{!}: H_{*}(B) \rightarrow H_{2 n_{F}+*}(P), \quad \alpha \mapsto \mathrm{PD}_{P}^{-1} \pi^{*} \mathrm{PD}_{B}(\alpha) .
$$

In other words, we consider cycles in $P$ that are either images of cycles in $F$ under $\iota$, or preimages under $\pi$ of cycles in $B$. Let $\iota_{C}$ denote the map in homology induced from the natural inclusion of $F$ in $P_{C}$. We have:

Theorem 2.4 [5, Theorem A] Let $\pi: P \rightarrow B$ be a Hamiltonian fibration with relative semipositive fiber $(F, \omega)$. Let $\sigma \in H_{2}(P, \mathbb{Z})$ and suppose $\sigma_{B}:=\pi_{*}(\sigma) \neq 0$ only admits simple decompositions for some $\omega_{B}$-tame almost complex structure $J_{B}$ in $B$. Let $c_{i}^{P}, c_{i}^{B}, c_{i}^{F}$ be as in ( $\star$ ). For generic fibered almost complex structure lifting $J_{B}$, we have

$$
\left\langle D ; c_{1}^{P}, \ldots, c_{l}^{P}\right\rangle_{\sigma, l}^{P}=\left\langle D ; c_{1}^{B}, \ldots, c_{l}^{B}\right\rangle_{\sigma_{B}, l}^{B} \cdot \sum_{\sigma^{\prime} \in B_{\sigma}}\left\langle[\mathrm{pt}] ; \iota_{C}\left(c_{1}^{F}\right), \ldots, \iota_{C}\left(c_{l}^{F}\right)\right\rangle_{\sigma^{\prime}, l}^{P_{C}},
$$

where $C$ is a curve counted in $\left\langle D ; c_{1}^{B}, \ldots, c_{l}^{B}\right\rangle_{\sigma_{B}, l}^{B}$ and $D \in H_{*}\left(\overline{\mathcal{M}}_{0, l}\right)$.

This formula in particular states that the sum in the righthand side of the formula is independent of the chosen $C$. Note that this sum is well-defined due to Gromov's compactness.

The core of the proof consists in establishing that the GW-invariants involved are generically and simultaneously well-defined, in other words the problem is to realize transversality while preserving the map $\bar{\pi}$ defined above. The proof of this is based on the relation

$$
\pi_{*} \circ D^{P}=D^{B} \circ \pi_{*},
$$

where $D^{P}$ and $D^{B}$ respectively stand for the Fredholm operators obtained by linearizing the Cauchy Riemann operators $\bar{\partial}_{J_{P}}$ and $\bar{\partial}_{J_{B}}$. As a consequence, we derive an 
exact sequence

$$
0 \rightarrow \operatorname{ker} D^{v} \rightarrow \operatorname{ker} D^{P} \rightarrow \operatorname{ker} D^{B} \rightarrow \operatorname{coker} D^{v} \rightarrow \operatorname{coker} D^{P} \rightarrow \operatorname{coker} D^{B} \rightarrow 0,
$$

where $D^{v}$ denotes the restriction of $D^{P}$ to vector fields along the curves that are vertically valued, ie with values in $\operatorname{ker} d \pi$. The vanishing, at least at the level of the universal moduli spaces, of the obstructions in the sequence above is provided by: (1) the irreducibility hypothesis on $\sigma_{B}$ for the vanishing of the last term of the sequence; (2) perturbing the Hamiltonian connection for the vanishing of coker $D^{v}$. It follows from standard arguments that for generic fibered almost complex structure the following holds:

- The subset $\mathcal{M}_{0, l}^{* *}\left(P, \sigma, J_{P}\right)$ of $\mathcal{M}_{0, l}\left(P, \sigma, J_{P}\right)$ consisting of simple maps that project to simple maps under $\bar{\pi}$ and the moduli space $\mathcal{M}_{0, l}^{*}\left(B, \sigma_{B}, J_{B}\right)$ are oriented manifolds.

- For countably many $(u, \mathbf{x}) \in \mathcal{M}_{0, l}^{*}\left(B, \sigma_{B}, J_{B}\right)$, the preimage $\bar{\pi}^{-1}(u, \mathbf{x})$ is an oriented manifold.

More generally, $\bar{\pi}$ extends to a map, using stabilization, between the compactifications $\overline{\mathcal{M}}_{0, l}\left(P, \sigma, J_{P}\right)$ and $\overline{\mathcal{M}}_{0, l}\left(B, \sigma_{B}, J_{B}\right)$. We can repeat the arguments above for each stratum in $\overline{\mathcal{M}}_{0, l}\left(P, \sigma, J_{P}\right)$ projecting to some stratum in $\overline{\mathcal{M}}_{0, l}\left(B, \sigma_{B}, J_{B}\right)$. The irreducibility hypothesis on the decompositions of $\sigma_{B}$ ensures that the coker $D^{B}$ term always vanishes and that the image under the evaluation map $\mathrm{ev}_{l, J_{B}}^{B}$ of the lower strata in $\overline{\mathcal{M}}_{0, l}\left(B, \sigma_{B}, J_{B}\right)$ have codimension at least 2 with respect to the top stratum $\mathcal{M}^{*}\left(B, \sigma_{B}, J_{B}\right)$. Furthermore, condition ( $\star$ ) ensures that the image under the evaluation map ev $P$, $J_{P}$ of the lower strata in $\overline{\mathcal{M}}_{0, l}\left(P, \sigma, J_{P}\right)$ have codimension at least two with respect to $\mathcal{M}_{0, l}^{* *}\left(P, \sigma, J_{P}\right)$. Once transversality is achieved, one recovers the formula using diagram (2-1) and the following observations:

- Since all cokernels vanish, it follows from the exact sequence above that

$$
\operatorname{det}\left(\operatorname{ker} D^{P}\right) \cong \operatorname{det}\left(\operatorname{ker} D^{B}\right) \otimes \operatorname{det}\left(\operatorname{ker} D^{v}\right) .
$$

Thus, the orientation of $\mathcal{M}_{0, l}^{* *}\left(P, \sigma, J_{P}\right)$ is given by the product of the orientations of $\mathcal{M}_{0, l}^{*}\left(B, \sigma_{B}, J_{B}\right)$ and $\bar{\pi}^{-1}\left(u_{B}, \mathbf{x}\right)$, where $\left(u_{B}, \mathbf{x}\right) \in \mathcal{M}_{0, l}^{*}\left(B, \sigma_{B}, J_{B}\right)$. Moreover, the orientations of the product pseudo-cycles are also given by a product

$$
\operatorname{det} T \mathcal{C}_{P} \cong \operatorname{det} T \mathcal{C}_{B} \otimes \operatorname{det} T \mathcal{C}_{F} .
$$

- It follows from symplectic triviality of $P$ over the 1 -skeleton of $B$, that for any two $J_{B}$-holomorphic maps representing the same class $\sigma_{B}$, the restrictions of $P$ to the images of the two maps are isomorphic as Hamiltonian fibrations. Hence, \langle\rangle$_{\sigma^{\prime}, l}^{P_{C}}$ does not depend on $C$. 
The proof is concluded by showing, using standard arguments, the independence with respect to the generic fibered almost complex structure of the Gromov-Witten invariants involved in the formula. See [5] for the details.

\subsection{Quantum homology and Seidel elements}

We start with the definition of (small) quantum homology with universal Novikov ring. As a module the quantum homology of $(X, \omega)$ is given by

$$
\mathrm{QH}_{*}(X) \equiv \mathrm{QH}_{*}(X, \Lambda):=H_{*}(X, \mathbb{Q}) \otimes \Lambda,
$$

where $\Lambda$ denotes some coefficient ring that we specify. We will take $\Lambda:=\Lambda^{\text {univ }}\left[q^{-1}, q\right]$ where $\Lambda^{\text {univ }}$ is the ring of generalized Laurent series in variable $t^{-1}$, ie an element $\lambda \in \Lambda^{\text {univ }}$ can be written as a formal sum

$$
\lambda=\sum_{i \geq 0} \lambda_{i} t^{r_{i}}, \quad \lambda_{i} \in \mathbb{Q}, r_{i} \in \mathbb{R}, \quad r_{i}>r_{i+1}, \quad \lim _{i \rightarrow \infty} r_{i}=-\infty .
$$

The grading on $\Lambda$ is given by imposing that $\operatorname{deg}(q)=2$. Let $\Lambda_{j}$ denote the set of elements of degree $2 j$ in $\Lambda$; then we give quantum homology the following grading:

$$
\mathrm{QH}_{k}(X):=\bigoplus_{i+2 j=k} H_{i}(X, \mathbb{Q}) \otimes \Lambda_{j}
$$

Next, we introduce the (small) quantum product

$$
\star: \mathrm{QH}_{i}(X) \otimes \mathrm{QH}_{j}(X) \rightarrow \mathrm{QH}_{i+j-2 n}(X), \quad(a, b) \mapsto a \star b .
$$

First, let $\left\{e_{v}\right\}$ be a basis of homology of $H_{*}(X)$, and let $\left\{e_{v}^{*}\right\}$ denote the corresponding dual basis with respect to the intersection pairing. Then, the quantum product is defined as follows: for $a \in H_{i}(X, \mathbb{Q})$ and $b \in H_{j}(X, \mathbb{Q})$, set

$$
a \star b=\sum_{B \in H_{2}^{S}(X), \nu}\left\langle a, b, e_{\nu}\right\rangle_{B, 3}^{X} e_{\nu}^{*} \otimes q^{-c_{1}(B)} t^{-\omega(B)} .
$$

We extend this by linearity with respect to $\Lambda$. Note that $[X]=1$ is the identity for this ring structure. Now, consider the $\Lambda$-submodule

$$
\mathcal{Q}_{-}:=\bigoplus_{i<2 n} H_{i}(X) \otimes \Lambda
$$

The following lemma of McDuff will be essential when proving Theorem 1.2. We give its proof for the reader's convenience: 
Lemma 2.5 [15, Lemma 2.1] If $(X, \omega)$ is not strongly uniruled then $\mathcal{Q}_{-}$is an ideal in $\mathrm{QH}_{*}(X)$. Furthermore, if $\mathcal{Q}_{-}$is an ideal $a \in \mathrm{QH}_{*}(X)$ is a unit, then there exists $x \in \mathcal{Q}_{-}$and $\lambda \neq 0$ in $\Lambda$ such that

$$
a=1 \otimes \lambda+x .
$$

Proof Let $c \in \mathrm{QH}_{*}(X)$ and assume by contradiction that there exists $b \in \mathcal{Q}_{-}$such that $c \star b \notin \mathcal{Q}_{-}$. By definition of the quantum product this means that there exists $B \in H_{2}^{S}(X)$ such that

$$
\langle c, b, \mathrm{pt}\rangle_{B, 3}^{X} \neq 0 .
$$

Hence $(X, \omega)$ is strongly uniruled which is impossible. Next, if $a$ is a unit, then $a \notin \mathcal{Q}_{-}$, for if it was we would have that 1 belongs to $\mathcal{Q}_{-}$. Therefore, we can write $a=1 \otimes \lambda+x$ with $x \in \mathcal{Q}_{-}$and $\lambda \neq 0$.

Seidel's representation This is a representation of $\pi_{1}(\operatorname{Ham}(X, \omega))$ in the subring $\mathrm{QH}_{*}^{\times}(X, \Lambda)$ of units of the quantum homology of $(X, \omega)$. Recall that $\pi: P_{\gamma} \rightarrow S^{2}$ denotes the Hamiltonian fibration obtained from $\gamma \in \pi_{1}(\operatorname{Ham}(X, \omega))$ via the clutching construction. Also, let $\mathcal{H}_{\gamma} \subset H_{2}^{S}\left(P_{\gamma}\right)$ denote the subset of section classes, ie of spherical classes that project to $\left[S^{2}\right]$ under the fibration projection.

Definition 2.4 The Seidel representation map

$$
S: \pi_{1}(\operatorname{Ham}(X, \omega)) \rightarrow \mathrm{QH}_{*}^{\times}(X, \Lambda), \quad \gamma \mapsto S(\gamma)
$$

is defined as

$$
S(\gamma):=\sum_{\sigma \in \mathcal{H}_{\gamma}, \nu}\left\langle\iota\left(e_{\nu}\right)\right\rangle_{\sigma, 1}^{P_{\gamma}} e_{\nu}^{*} \otimes q^{-c_{\gamma}(\sigma)} t^{-\tau_{\gamma}(\sigma)} .
$$

Geometrically, $S(\gamma)$ "counts" holomorphic sections of $P_{\gamma}$ intersecting the cycles $e_{\mu}$ in the fiber above the north pole of $S^{2}$. The splitting axiom for fibrations over $S^{2}$ [17, Theorem 11.4.1] gives that for all section classes $\sigma \in H_{2}^{S}\left(P_{\gamma}\right)$ and for classes $\alpha_{1}, \ldots, \alpha_{l} \in H_{*}(F)$ the following holds for every integer $0 \leq k \leq l$ :

$$
\begin{array}{r}
\left\langle[\mathrm{pt}] ; \iota\left(\alpha_{1}\right), \ldots, \iota\left(\alpha_{l}\right)\right\rangle_{\sigma, l}^{P_{\gamma}}=\sum_{A \in H_{2}^{S}(F ; \mathbb{Z}), \nu}\left\langle[\mathrm{pt}] ; \iota\left(\alpha_{1}\right), \ldots, \iota\left(\alpha_{k}\right), \iota\left(e_{v}\right)\right\rangle_{\sigma-\iota(A), k+1}^{P_{\gamma}} \\
\cdot\left\langle[\mathrm{pt}] ; e_{v}^{*}, \alpha_{k+1}, \ldots, \alpha_{l}\right\rangle_{A, l-k+1}^{F}
\end{array}
$$

It follows easily that the $\Lambda$ linear action of $S(\gamma)$ on $a \in H_{*}(F)$ is given by

$$
S(\gamma)(a):=S(\gamma) \star a=\sum_{\sigma \in \mathcal{H}_{\gamma}, \nu}\left\langle\iota(a), \iota\left(e_{\nu}\right)\right\rangle_{\sigma, 2}^{P_{\gamma}} e_{\nu}^{*} \otimes q^{-c_{\gamma}(\sigma)} t^{-\tau_{\gamma}(\sigma)} .
$$


Note that in the above notation $S(\gamma)=S(\gamma)$ (1). Let us define the following equivalence class on section classes: we say that $\sigma_{1}$ is equivalent to $\sigma_{2}$ if and only if

$$
\tau_{\gamma}\left(\sigma_{1}-\sigma_{2}\right)=0=c_{\gamma}\left(\sigma_{1}-\sigma_{2}\right) .
$$

We will use the notation $[\sigma]$ to denote the equivalence class of $\sigma$. From $[10$, Theorem 3.A] we have:

Lemma 2.6 For every $\gamma \in \pi_{1}(\operatorname{Ham}(F, \omega))$ and every nonzero $a \in H_{*}(F)$, there exists an equivalence class of section classes $[\sigma]$ and $b \in H_{*}(F)$ such that

$$
\sum_{\sigma^{\prime} \in[\sigma]}\langle\iota(a), \iota(b)\rangle_{2, \sigma^{\prime}}^{P_{\gamma}} \neq 0 .
$$

In particular there is a section class $\sigma \in \mathcal{H}_{\gamma}$ such that $\langle\iota(a), \iota(b)\rangle_{2, \sigma}^{\boldsymbol{P}_{\gamma}} \neq 0$.

Proof Assume it is not true. Equation (2-4) and linearity of Gromov-Witten invariants then imply that $S(\gamma)(a)=0$. But since $S(\gamma)$ is invertible, this is only possible for $a=0$; hence the contradiction.

\section{Proofs of the results}

We are now ready to prove the results. We begin by proving Theorem 1.2 and Theorem 1.5. More precisely, we prove the following:

Theorem 3.1 Assume $(F, \omega)$ verifies $(\star)$ and is not strongly uniruled. If $\left(B, \omega_{B}\right)$ is symplectically uniruled for some class $\sigma_{B}$ admitting only simple decompositions, then $P$ is also symplectically uniruled. Moreover, if $[\mathrm{pt}]_{Q}^{l} \neq 0$, and if $\left(B, \omega_{B}\right)$ is $(l+1)-S R C$ for $\sigma_{B}$ admitting only simple decompositions, then $P$ is at least $l-S R C$.

Proof By assumption there exists a nonzero $\sigma_{B} \in H_{2}^{S}(B)$ and classes $c_{1}^{B}, \ldots, c_{l}^{B} \in$ $H_{*}(B)$ such that

$$
\left\langle D ;[\mathrm{pt}], c_{1}^{B}, \ldots, c_{l}^{B}\right\rangle_{\sigma_{B}, l+1}^{B} \neq 0 .
$$

Let $C$ be the image of a map counted in (3-1). Since $\sigma_{B} \neq 0$, the restriction $P_{C}$ of $P$ to $C$ is a Hamiltonian fibration over $S^{2}$. Let $\phi \in \pi_{1}(\operatorname{Ham}(F, \omega))$ be a Hamiltonian loop corresponding to this fibration, and let $S(\phi) \in \mathrm{QH}^{\times}(F ; \Lambda)$ denote the corresponding Seidel element. Since $(F, \omega)$ is not strongly uniruled, it follows from Lemma 2.5 that there exists $0 \neq \lambda \in \Lambda$ and $x \in \mathcal{Q}_{-}$such that

$$
S(\phi)=1 \otimes \lambda+x .
$$


In particular, this directly implies that there is an equivalence class $[\sigma]$ of section classes in $P_{C}$ such that

$$
0 \neq \sum_{\sigma^{\prime} \in[\sigma]}\langle[\mathrm{pt}]\rangle_{\sigma^{\prime}, 1}^{P_{C}}=\sum_{\sigma^{\prime} \in[\sigma]}\left\langle[\mathrm{pt}] ;[\mathrm{pt}], \iota_{C}([F]), \ldots, \iota_{C}([F])\right\rangle_{\sigma^{\prime}, l+1}^{P_{C}} .
$$

Let $\iota_{P_{C}}$ denote the inclusion in homology induced by the inclusion of $P_{C}$ into $P$. It is easy to see that the image under $\iota_{P_{C}}$ of $[\sigma]$ defines an equivalence class of spherical classes projecting on $\sigma_{B}$, in the sense that for any $\sigma_{1}, \sigma_{2} \in \iota_{P_{C}}([\sigma])$ we have

$$
\tau\left(\sigma_{1}-\sigma_{2}\right)=0=c_{v}\left(\sigma_{1}-\sigma_{2}\right) .
$$

By the product formula for Gromov-Witten invariants (2-2),

$$
\begin{aligned}
& \sum_{\sigma^{\prime} \in l_{P_{C}}([\sigma])}\left\langle D ;[\mathrm{pt}], \pi^{!}\left(c_{1}^{B}\right), \ldots, \pi^{!}\left(c_{l}^{B}\right)\right\rangle_{\sigma^{\prime}, l+1}^{P} \\
= & \sum_{\sigma^{\prime} \in \iota_{P_{C}}}\langle[\sigma]) \\
= & \left\langle D ;[\mathrm{pt}], c_{1}^{B}, \ldots, c_{l}^{B}\right\rangle_{\sigma_{B}, l+1}^{B} \sum_{\sigma^{\prime} \in[\sigma]}\left\langle[\mathrm{pt}] ;[\mathrm{pt}], \iota_{C}([F]), \ldots, c_{l}^{B}\right\rangle_{\sigma_{B}, l+1}^{B} \sum_{\sigma^{\prime \prime} \in B_{\sigma^{\prime}}}\left\langle[\mathrm{pt}] ;[\mathrm{pt}], \iota_{C}([F]), \ldots, \iota_{C}([F])\right\rangle_{\sigma^{\prime \prime}, l+1}^{P_{C}}\left([F\rangle_{\sigma^{\prime}, l+1}^{P_{C}} \neq 0 .\right.
\end{aligned}
$$

In particular, there is at least one $\sigma^{\prime} \in \iota_{P_{C}}([\sigma])$ such that

$$
\left\langle D ;[\mathrm{pt}], \pi^{!}\left(c_{1}^{B}\right), \ldots, \pi^{!}\left(c_{l}^{B}\right)\right\rangle_{\sigma^{\prime}, l+1}^{P} \neq 0 .
$$

Now we prove the second assertion of the theorem. Since for every loop $\phi$ of Hamiltonian diffeomorphisms of $F$ the Seidel element $S(\phi)$ is an unit in $\mathrm{QH}_{*}(F ; \Lambda)$, we have that

$$
0 \neq S(\phi) \star\left([\mathrm{pt}]_{Q}^{l}\right) .
$$

Using the splitting axiom for Gromov-Witten invariants, and from the definition of the Seidel element, one obtains that

$$
\begin{aligned}
S(\phi) \star\left([\mathrm{pt}]_{Q}^{l}\right) & =\sum_{A \in H_{2}^{S}(F), \nu}\left\langle[\mathrm{pt}] ;[\mathrm{pt}], \ldots,[\mathrm{pt}], e_{\nu}\right\rangle_{A, l+1}^{F} S(\phi)\left(e_{\nu}^{*}\right) \otimes q^{-c_{1}^{T F}(A)} t^{-\omega(A)} \\
& =\sum_{\sigma \in \mathcal{H}_{\phi}, A \in H_{2}^{S}(F), \nu, \mu}\left\langle[\mathrm{pt}] ;[\mathrm{pt}], \ldots,[\mathrm{pt}], e_{\nu}\right\rangle_{A, l+1}^{F}\left\langle\iota\left(e_{\nu}^{*}\right), \iota\left(e_{\mu}\right)\right\rangle_{\sigma, 2}^{P_{\phi}} \\
& =\sum_{\tilde{\sigma} \in \mathcal{H}_{\phi}, \mu}\left\langle[\mathrm{pt}] ;[\mathrm{pt}], \ldots,[\mathrm{pt}], \iota\left(e_{\mu}\right)\right\rangle_{\widetilde{\sigma}, l+1}^{P_{\phi}} \cdot e_{\mu}^{*} \otimes q^{-c_{\phi}(\sigma+\iota(A))} t^{-\tau_{\phi}(\sigma+\iota(A))}
\end{aligned}
$$


Hence, there is a class $a \in H_{*}(F)$ and a class of section classes $[\sigma]$ such that

$$
\sum_{\sigma^{\prime} \in[\sigma]}\langle[\mathrm{pt}] ;[\mathrm{pt}], \ldots,[\mathrm{pt}], \iota(a)\rangle_{\sigma^{\prime}, l+1}^{P_{\phi}} \neq 0 .
$$

We conclude by the use of the product formula as before.

Now Theorem 1.2 follows easily:

Proof of Theorem 1.2 In case where $(F, \omega)$ is strongly uniruled, the conclusion follows from Theorem 1.1. Otherwise, we simply use Theorem 3.1 to conclude.

As for Theorem 1.2, Corollary 1.3 follows directly from Theorem 1.1 and the proposition below. This latter proposition is a simple consequence of Theorem 2.1 asserting that projectively uniruled manifolds are strongly symplectically uniruled.

Proposition 3.2 Assume $(F, \omega)$ verifies ( $\star$ ) and is not strongly uniruled. Also assume that $\left(B, J_{B}, \omega_{B}\right)$ is a uniruled projective manifold. Then $P$ is $S S U$.

Proof From Lemma 2.2, $\left(B, J_{B}, \omega_{B}\right)$ is uniruled for a class $\sigma_{B}$ verifying the assumptions of Theorem 3.1, and which is obstruction free. Hence, the product formula can be applied and the proof follows.

\section{References}

[1] A Blanchard, Sur les variétés analytiques complexes, Ann. Sci. Ecole Norm. Sup. 73 (1956) 157-202 MR0087184

[2] B Chen, A-M Li, Symplectic virtual localization of Gromov-Witten invariants arXiv: math/0610370

[3] H Hofer, C Viterbo, The Weinstein conjecture in the presence of holomorphic spheres, Comm. Pure Appl. Math. 45 (1992) 583-622 MR1162367

[4] J Hu, T-J Li, Y Ruan, Birational cobordism invariance of uniruled symplectic manifolds, Invent. Math. 172 (2008) 231-275 MR2390285

[5] C Hyvrier, A product formula for Gromov-Witten invariants, to appear in J. Symplectic Geom. arXiv:0904.1492

[6] J Kẹdra, Restrictions on symplectic fibrations, Differential Geom. Appl. 21 (2004) 93-112 MR2067461 With an appendix by the author and K Ono

[7] J Kollár, Rational curves on algebraic varieties, Ergeb. Math. Grenzgeb. 32, Springer, Berlin (1996) MR1440180 
[8] J Kollár, Low degree polynomial equations: arithmetic, geometry and topology, from: "European Congress of Mathematics, Vol. I (Budapest, 1996)", Progr. Math. 168, Birkhäuser, Basel (1998) 255-288 MR1645812

[9] F Lalonde, D McDuff, Symplectic structures on fiber bundles, Topology 42 (2003) 309-347 MR1941438

[10] F Lalonde, D McDuff, L Polterovich, Topological rigidity of Hamiltonian loops and quantum homology, Invent. Math. 135 (1999) 369-385 MR1666763

[11] J Li, G Tian, Virtual moduli cycles and Gromov-Witten invariants of general symplectic manifolds, from: "Topics in symplectic 4-manifolds (Irvine, CA, 1996)", (R J Stern, editor), First Int. Press Lect. Ser. I, Int. Press, Cambridge, MA (1998) 47-83 MR1635695

[12] T Li, Y Ruan, Uniruled symplectic divisors arXiv:0711.4254

[13] G Liu, G Tian, Weinstein conjecture and GW-invariants, Commun. Contemp. Math. 2 (2000) 405-459 MR1806943

[14] G Lu, The Weinstein conjecture in the uniruled manifolds, Math. Res. Lett. 7 (2000) 383-387 MR1783615

[15] D McDuff, Hamiltonian $S^{1}$-manifolds are uniruled, Duke Math. J. 146 (2009) 449507 MR2484280

[16] D McDuff, D Salamon, Introduction to symplectic topology, second edition, Oxford Math. Monogr., The Clarendon Press, Oxford Univ. Press, New York (1998) MR1698616

[17] D McDuff, D Salamon, J-holomorphic curves and symplectic topology, Amer. Math. Soc. Colloquium Publ. 52, Amer. Math. Soc. (2004) MR2045629

[18] Y Ruan, Virtual neighborhoods and pseudo-holomorphic curves, from: "Proceedings of 6th Gökova Geometry-Topology Conference”, 23 (1999) 161-231 MR1701645

[19] Y Ruan, G Tian, A mathematical theory of quantum cohomology, J. Differential Geom. 42 (1995) 259-367 MR1366548

[20] P Seidel, $\pi_{1}$ of symplectic automorphism groups and invertibles in quantum homology rings, Geom. Funct. Anal. 7 (1997) 1046-1095 MR1487754

[21] C Viterbo, A proof of Weinstein's conjecture in $\mathbf{R}^{2 n}$, Ann. Inst. H. Poincaré Anal. Non Linéaire 4 (1987) 337-356 MR917741

[22] A Weinstein, On the hypotheses of Rabinowitz' periodic orbit theorems, J. Differential Equations 33 (1979) 353-358 MR543704

Mathematics Department, Uppsala Universitet

Box 480, SE-75106 Uppsala, Sweden

clement.hyvrier@math.uu.se

Received: 6 April $2011 \quad$ Revised: 17 February 2012 